Document downloaded from:

http://hdl.handle.net/10251/98071

This paper must be cited as:

De Rosario Martínez, H.; Page Del Pozo, AF.; Mata Amela, V. (2014). Point of optimal kinematic error: Improvement of the instantaneous helical pivot method for locating centers of rotation. Journal of Biomechanics. 47(7):1742-1747. doi:10.1016/j.jbiomech.2014.02.003

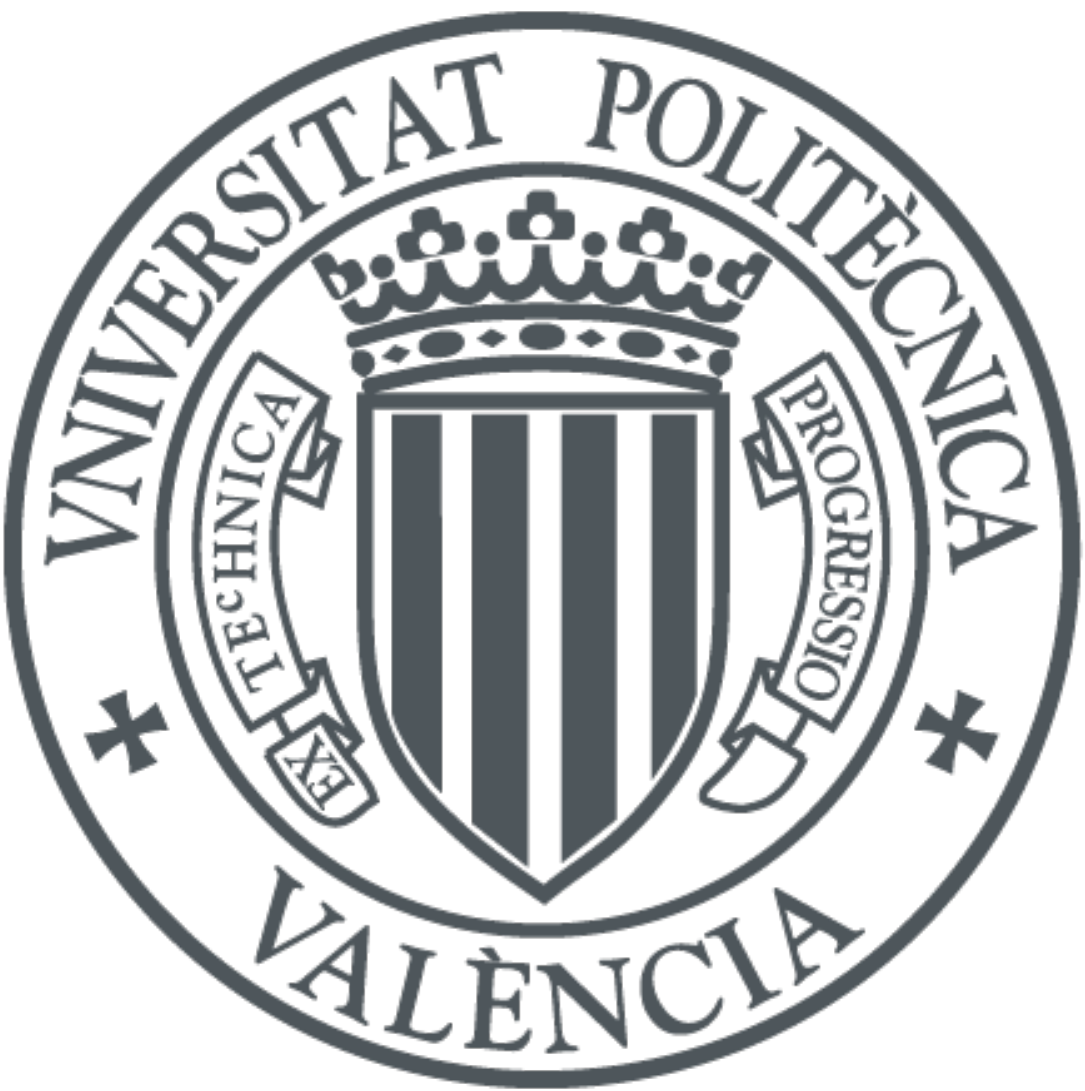

The final publication is available at

http://dx.doi.org/10.1016/j.jbiomech.2014.02.003

Copyright Elsevier

Additional Information 


\section{Point of optimal kinematic error: improvement of the instantaneous helical pivot method for locating centers of rotation}

Helios De Rosario ${ }^{\mathrm{a}, \mathrm{c}, *}$, Álvaro Page ${ }^{\mathrm{b}, \mathrm{c}}$, Vicente Mata ${ }^{\mathrm{d}}$

a Instituto de Biomecánica de València, Valencia, Spain

${ }^{\mathrm{b}}$ Departamento de Física Aplicada. Universitat Politècnica de València.

${ }^{\mathrm{c}}$ Grupo de Tecnología Sanitaria del IBV, CIBER de Bioingeniería, Biomateriales y Nanomedicina (CIBER-BBN), Valencia, Spain

d Departamento de Ingeniería Mecánica y de Materiales. Universitat Politècnica de València.

* Corresponding author: Instituto de Biomecánica de Valencia, Universitat Politècnica de Valencia, Edificio 9C, Camino de Vera s/n, E-46022, Valencia, Spain. Tel: +34 963879160. Fax: +34 963879169. E-mail: helios.derosario@ibv.upv.es

Keywords: center of rotation, instantaneous helical axis, functional calibration, optimization

DOI: $\underline{10.1016 / j . j b i o m e c h .2014 .02 .003}$

(C) 2014, Helios De Rosario, Álvaro Page \& Vicente Mata. This manuscript version is made available under the CC-BY-NC-ND 4.0 license http://creativecommons.org/licenses/by-nc-nd/4.0/

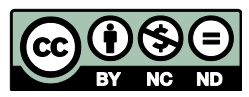




\begin{abstract}
This paper proposes a variation of the instantaneous helical pivot technique for locating centers of rotation. The point of optimal kinematic error (POKE), which minimizes the velocity at the center of rotation, may be obtained by just adding a weighting factor equal to the square of angular velocity in Woltring's equation of the pivot of instantaneous helical axes (PIHA). Calculations are simplified with respect to the original method, since it is not necessary to make explicit calculations of the helical axis, and the effect of accidental errors is reduced. The improved performance of this method was validated by simulations based on a functional calibration task for the gleno-humeral joint center. Noisy data caused a systematic dislocation of the calculated center of rotation towards the center of the arm marker cluster. This error in PIHA could even exceed the effect of soft tissue artifacts associated to small and medium deformations, but it was successfully reduced by the POKE estimation.
\end{abstract}




\section{Introduction}

A well known method for locating the center of rotation $(\mathrm{CoR})$ of a ball joint consists of calculating the "pivot" point of the instantaneous helical axes (IHA) of a set of calibration gestures. This method was first proposed by Woltring (1990), and it is still very used, specially for the gleno-humeral joint after the recommendation of the International Society of Biomechanics (Wu et al., 2005). The conventional procedure consists of three steps. First, calculate the instantaneous kinematic parameters of the relative motion between the linked segments, defined by the angular velocity $\left(\boldsymbol{\omega}_{t}\right)$ and the velocity of an arbitrary point $\mathbf{p}_{t}\left(\dot{\mathbf{p}}_{t}\right)$, for each instant $t=1, \ldots, n$. Second, calculate IHA positions as:

$$
\mathbf{S}_{t}=\dot{\mathbf{p}}_{t}+\frac{\boldsymbol{\omega}_{t} \times \dot{\mathbf{p}}_{t}}{\omega_{t}^{2}}
$$

And third, calculate the pivot of IHA (PIHA) by solving the following equation:

$$
\left(\sum_{t=1}^{n} \mathbf{Q}_{t}\right) \mathbf{s}_{P I H A}=\sum_{t=1}^{n} \mathbf{Q}_{t} \mathbf{s}_{t}
$$

where $\mathbf{Q}_{t}$ are orthogonal projection matrices, defined by the unit vectors of $\boldsymbol{\omega}_{t}\left(\mathbf{n}_{t}\right)$ and the identity matrix $\mathbf{I}$ :

$$
\mathbf{Q}_{t}=\mathbf{I}-\mathbf{n}_{t} \mathbf{n}_{t}^{T}
$$

This "averaging" procedure cancels out IHA errors that present opposing directions during the calibration movements. However, its precision is challenged by the high sensitivity of IHA errors to low angular velocities. This may be solved by discarding all the instants where $\omega_{t}$ is below a threshold, often set at $0.25 \mathrm{rad} / \mathrm{s}$ for measures taken between 10 and $50 \mathrm{~Hz}$ (Monnet et al., 2007; Stokdijk et al., 2000; Veeger and Yu, 1996).

An alternative proposed by Halvorsen et al. (1999) consists of calculating the pivot of the finite helical axis (FHA), which defines the locus of minimum displacement from a reference position (Woltring et al., 1985). This variant has become very used too, and its numerical properties have been studied in detail. FHA are very sensitive to small rotations (instead of small velocities), but this is normally solved by including a 
weighting factor $w_{t}$ (do not confound with angular velocities) depending on the rotation angle $\vartheta_{t}$. An optimal solution has been found in $w_{t}=\sin ^{2}\left(\vartheta_{t} / 2\right)$, which gives the minimum error in terms of CoR displacement (Ehrig et al., 2006).

In this paper we propose a similar optimization of the PIHA method, which manages more effectively the sensitivity of IHA errors to angular velocities. This hypothesis was validated by a simulation, modeled upon a real measurement of the gleno-humeral joint.

\section{Materials and methods}

\subsection{Optimization of the PIHA method}

Like its variant based on FHA, Woltring's method may be optimized by adding a weighting factor equal to $\omega_{t}^{2}$, which ensures the minimum error in terms of relative velocity in the CoR (see Appendix A.1). Thus, (2) may be rewritten to give the point of optimal kinematic error (POKE):

$$
\left(\sum_{t=1}^{n} \omega_{t}^{2} \mathbf{Q}_{t}\right) \mathbf{S}_{\text {POKE }}=\sum_{t=1}^{n} \omega_{t}^{2} \mathbf{Q}_{t} \mathbf{s}_{t}
$$

This equation may be simplified, avoiding the explicit calculation of IHA, by setting the reference point $\mathbf{p}_{t}$ at the origin of coordinates for any $t$, and scaling $\mathbf{Q}_{t}$ by $\omega_{t}^{2}$ :

$$
\mathbf{W}_{t}=\omega_{t}^{2} \mathbf{Q}_{t}=\omega_{t}^{2} \mathbf{I}-\boldsymbol{\omega}_{t} \boldsymbol{\omega}_{t}^{T}
$$

Then, (4) becomes:

$$
\left(\sum_{t=1}^{n} \mathbf{W}_{t}\right) \mathbf{S}_{P O K E}=\sum_{t=1}^{n} \boldsymbol{\omega}_{t} \times \dot{\mathbf{p}}_{t}
$$

\subsection{Experimental validation}

A subject signed an informed consent to participate in the experiment. He was instrumented with three markers placed on the right acromion to measure the scapular motion (Karduna et al., 2001), and three markers on the skin of the upper arm. Arm markers had its center at $150 \mathrm{~mm}$ from the acromion, and they were separated about $110 \mathrm{~mm}$ from each other, although those distances varied due to STA. The subject made five consecutive cycles of typical functional calibration gestures: arm flexion-extension, 
elevation, and half-circumduction, with a maximum elevation of $45^{\circ}$ (Leardini et al., 1999).

These motions were recorded by 10 cameras at $50 \mathrm{~Hz}$, with a photogrammetry system (Kinescan/IBV). The rigid rotations of the humerus w.r.t. the scapula and the deformation of the humeral marker cluster were extracted from these measures, as by De Rosario et al. (2012). The ranges of marker displacements within the bone frame are presented in table 1. All measures were defined in local coordinates systems, that were aligned with the global reference frame (Wu and Cavanagh, 1995) when the subject adopted the reference posture (upright, arms at sides and palms facing forward).

A theoretical motion of the humeral markers was then simulated, repeating the measured rotation patterns, and assuming a joint center at $(40,-40,-10) \mathrm{mm}$ from the acromion, based on Stokdijk et al. (2000). That "ideal" center was used as reference point $\mathbf{p}_{t}$, so that the calculated centers would measure the CoR errors. The ideal motion was altered by a continuous noise based on Begon's et al. model (2007): marker positions were modeled as Gaussian functions of the motion cycle to simulate soft tissue artifacts (STA), disturbed by white noise (see Appendix A.2). STA were defined from the values of table 1, scaled by a factor equal to 0 (null artifact), 0.5 (small artifacts, with maximal marker displacements around $4.5 \mathrm{~mm}$ ), or 1 (medium artifacts, with maximum displacements around $9.0 \mathrm{~mm})$. The standard deviation of white noise $(\sigma)$ ranged from 0 to $1 \mathrm{~mm}$, in $0.1 \mathrm{~mm}$ steps.

Table 1. Maximum and minimum marker displacements by deformation (in $\mathrm{mm}$ ), for markers M1, M2, M3, in the three coordinates of the humerus frame. The maximum absolute values of each range are underlined.

\begin{tabular}{|c|c|c|c|c|c|c|c|c|c|c|}
\hline & \multicolumn{3}{|c|}{ M1 } & \multicolumn{3}{|c|}{ M2 } & \multicolumn{3}{|c|}{ M3 } \\
\hline & & $x$ & $y$ & $z$ & $x$ & $y$ & $z$ & $x$ & $y$ & $z$ \\
\hline \multirow[t]{2}{*}{ Fl.-Ext. } & $\min$ & -1.1 & -3.7 & $\underline{-3.5}$ & -0.5 & -2.9 & $\underline{-2.8}$ & $\underline{-1.4}$ & -1.7 & -3.5 \\
\hline & $\max$ & 1.6 & $\underline{4.0}$ & 2.4 & 1.0 & $\underline{4.4}$ & 2.2 & 1.2 & 1.3 & 4.8 \\
\hline \multirow[t]{2}{*}{ Elev. } & $\min$ & -0.5 & $\underline{-2.4}$ & $\underline{-6.0}$ & $\underline{-1.9}$ & -1.1 & -1.4 & $\underline{-0.7}$ & -3.7 & -1.0 \\
\hline & $\max$ & $\underline{2.0}$ & 1.5 & 1.0 & 0.5 & $\underline{4.9}$ & $\underline{3.5}$ & 0.3 & 0.5 & $\underline{3.0}$ \\
\hline \multirow[t]{2}{*}{ Half Circ. } & $\min$ & -1.3 & -5.6 & -6.4 & -1.4 & -3.4 & -2.0 & -1.8 & -2.8 & -3.6 \\
\hline & $\max$ & 2.8 & 4.1 & 2.0 & 0.8 & 7.4 & $\underline{3.1}$ & 1.2 & 0.3 & 6.9 \\
\hline
\end{tabular}

Fl.-Ext: flexion extension. Elev.: elevation. Half. Circ.: half circumduction. 
Each combination of STA and noise sizes was simulated 100 times. Marker positions and velocities were calculated from noisy data by a local polynomial filter of 7 th order. The filter's bandwidth was $N=13$ samples for an optimal calculation of velocities, considering that the marker motion was bandlimited below $5 \mathrm{~Hz}$, i.e. 0.1 times the sampling frequency (Lanshammar, 1982).

Marker positions and velocities $\left(\mathbf{r}_{i t}, \dot{\mathbf{r}}_{i t}\right)$ were used to calculate the kinematics of the arm (Page et al., 2009):

$$
\begin{gathered}
\mathbf{g}_{t}=\frac{1}{3} \sum_{i=1}^{3} \mathbf{r}_{i t} \quad ; \quad \dot{\mathbf{g}}_{t}=\frac{1}{3} \sum_{i=1}^{3} \dot{\mathbf{r}}_{i t} \\
\mathbf{J}_{t}=\sum_{i=1}^{3}\left(\left|\mathbf{r}_{i t}-\mathbf{g}_{t}\right|^{2} \mathbf{I}-\left(\mathbf{r}_{i t}-\mathbf{g}_{t}\right)\left(\mathbf{r}_{i t}-\mathbf{g}_{t}\right)^{T}\right) \\
\boldsymbol{\omega}_{t}=\mathbf{J}_{t}^{-1} \sum_{t=1}^{n}\left(\mathbf{r}_{i t}-\mathbf{g}_{t}\right) \times \dot{\mathbf{r}}_{i t} \\
\dot{\mathbf{p}}_{t}=\dot{\mathbf{g}}_{t}+\boldsymbol{\omega}_{t} \times\left(\mathbf{p}_{t}-\mathbf{g}_{t}\right)
\end{gathered}
$$

Then, PIHA and POKE estimates of the rotation centers were obtained, and their distances to the real CoR were compared.

Since random noise causes IHA errors proportional to noise amplitude (Page et al., 2007), the CoR error norms $(|\delta \mathbf{s}|)$ were fitted by a generalized linear model to a gamma distribution, with expected value proportional to $\sigma$, and different parameters for each STA scale $i$ :

$$
E(|\delta \mathbf{s}|)=\alpha_{i}+\beta_{i} \sigma
$$

The differences between PIHA and POKE errors $\left(|\delta \mathbf{s}|_{\text {PIHA }}-|\delta \mathbf{s}|_{\text {POKE }}\right)$ were fitted by a weighted linear model with the same equation. The weights of this model were chosen according to the observed variances of the different "continuous noise" models.

Then, the simple main effects of STA and their interaction with noise were statistically tested. All calculations were done with GNU Octave and R (Eaton et al., 2008; R Core Team, 2013). 


\section{Results}

For null artifacts, the error increased monotonically with noise amplitude, such that the calculated CoR was drawn off its real position towards the center of the marker cluster. However, this error was substantially reduced by the POKE method (see figure 1).

Figure 2 represents the average coordinates of the calculated centers for all STA scales, and show that the biasing effect of noise was kept in PIHA estimates, whereas POKE reduced that bias to the scale of medium-sized STA.

Figure 3 shows the confidence intervals of PIHA and POKE errors. For non-null STA, such errors were dominated by the artifact, although marker noise was still noticeable. On the other hand, the variability of such errors mainly depended on the STA scale. The inverses of these variances were used as weighting factors in the statistical analysis.

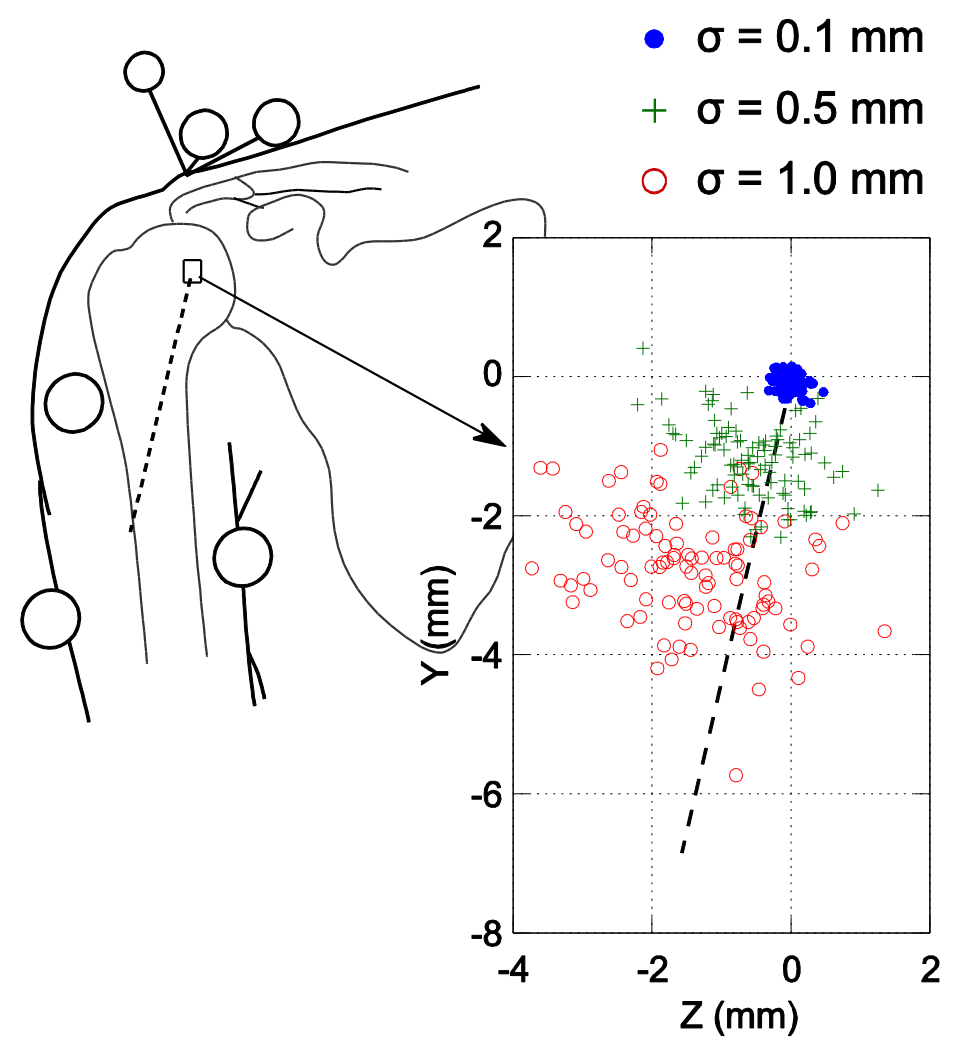

PIHA

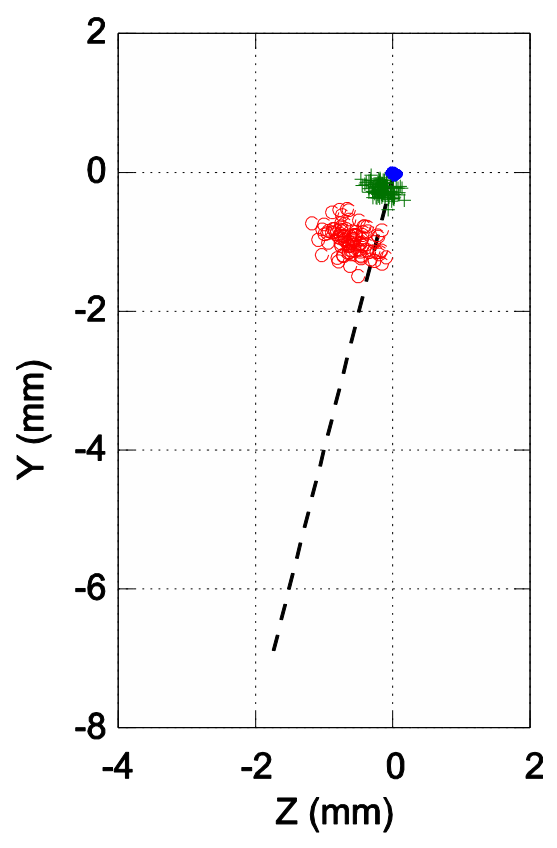

POKE

Figure 1. CoR errors in the YZ-plane, for null STA and three different noise levels. The dashed black line represents the separation between the error-free CoR and the center of the marker set. The measures inside the plot frame (in $\mathrm{mm}$ ) are exact; the sketch of the subject's shoulder and upper arm is an approximate representation to provide a visual clue of the proportions. 

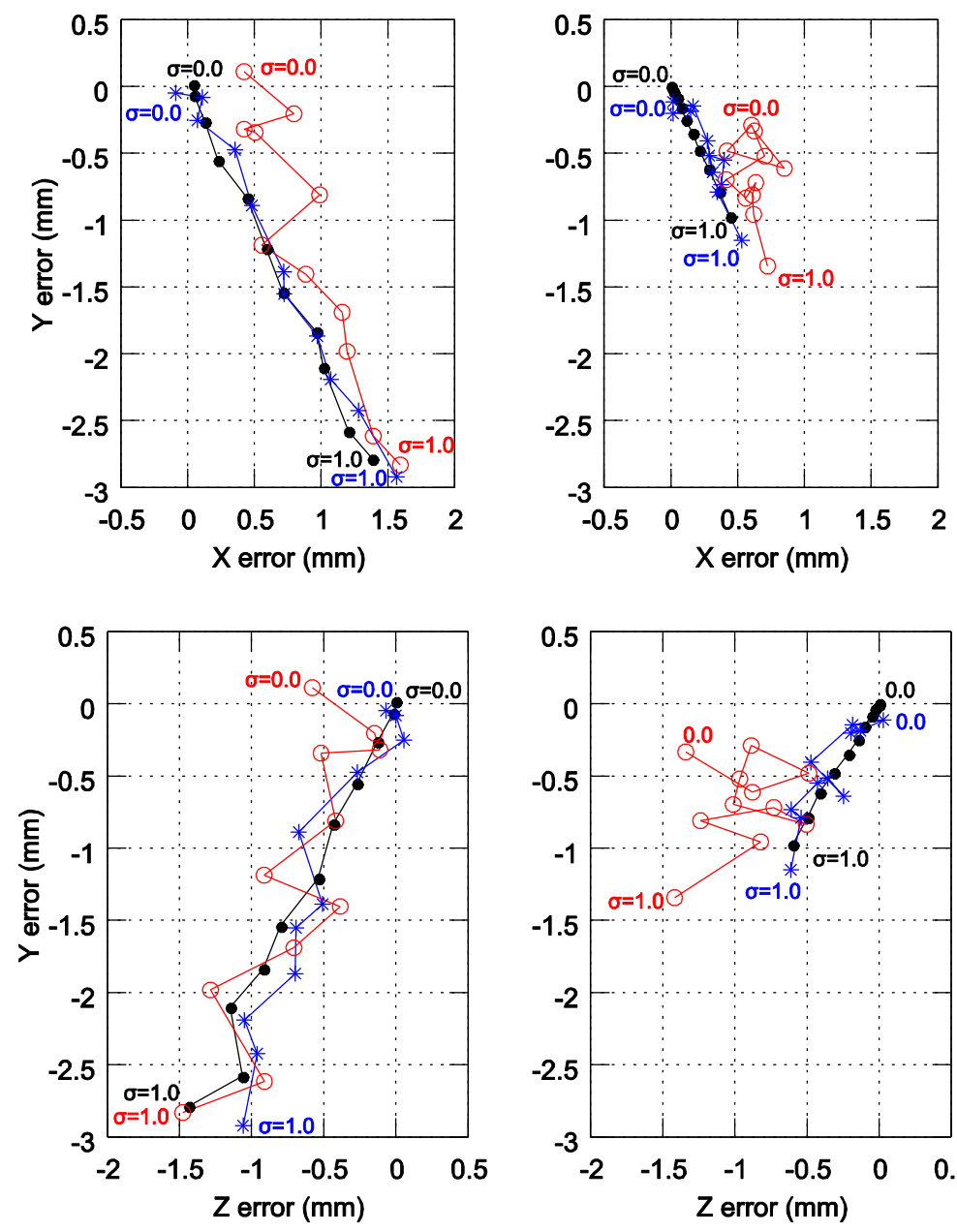

PIHA

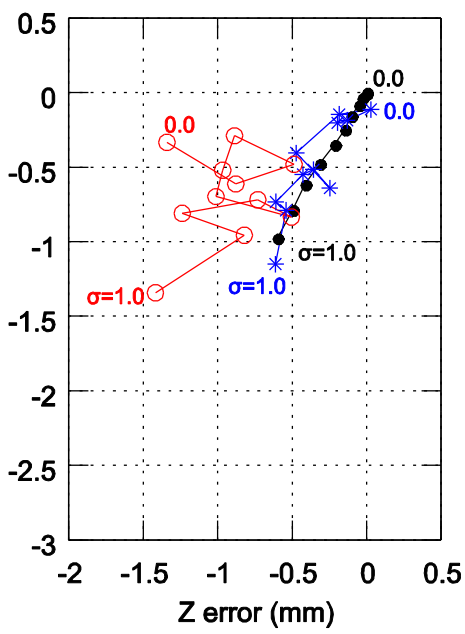

POKE

Figure 2. Average coordinates of calculated CoR with respect to the ideal rotation center, for all combinations of STA (different lines) and noise (different points within lines). Each line connects the increasing noise levels for each STA scale. The lowest and highest values of noise, $\sigma=0.0 \mathrm{~mm}$ and $\sigma=1.0 \mathrm{~mm}$, are explicitly labeled in each line. 


$$
\text { I Null STA }(\mathrm{x} 0) \quad \text { F Small STA }(\mathrm{x} 0.5) \quad \text { I Medium STA }(\mathrm{x} 1)
$$
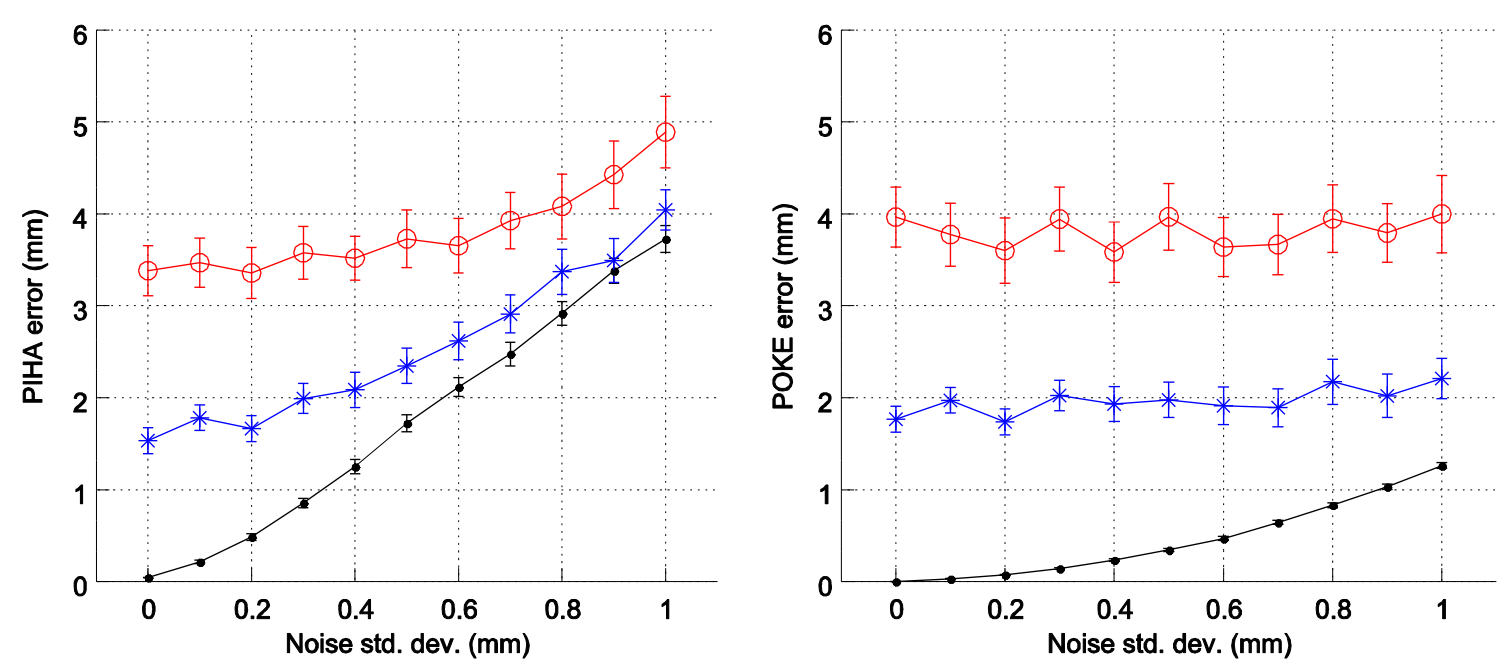

Figure 3. Average and 95\% confidence interval of the CoR mean error, measured as the distance between the "true" and measured CoR. Values are given for all combinations of STA and noise (100 measures per combination).

PIHA amplified the random noise in all cases (linear effects greater than $1 \mathrm{~mm} / \mathrm{mm}$, see table 2), unlike POKE. In fact, noise effects were statistically insignificant for mediumscaled STA with POKE.

The expected differences between PIHA and POKE errors, and the effect of noise on those differences, were positive in all cases (table 3), i.e. detrimental for Woltring's original method. STA masked the average differences (they were insignificant for medium-scaled STA), but not the effect of noise on them (over $1 \mathrm{~mm} / \mathrm{mm}$ for all STA scales).

Table 2. Linear effects of white noise on the CoR error $(\mathrm{mm} / \mathrm{mm})$ for PIHA and POKE, at fixed STA sizes. $F$-tests of simple effects (De Rosario, 2013; Fox and Weisberg, 2011), with $p$-values adjusted by Bonferroni's correction.

\begin{tabular}{llccrr} 
& STA scale & Value $(\mathrm{mm} / \mathrm{mm})$ & Std. error $(\mathrm{mm} / \mathrm{mm})$ & $F(1,3294)^{*}$ & $p$-value \\
\hline PIHA & null & 3.1313 & 0.0391 & 6417.979 & 0.000 \\
& small & 2.1922 & 0.0859 & 654.317 & 0.000 \\
& medium & 1.2108 & 0.1366 & 78.622 & 0.000 \\
& & & & \\
POKE & null & 0.75308 & 0.01088 & 4795.2697 & 0.000 \\
& small & 0.30759 & 0.08477 & 13.1671 & 0.002 \\
& medium & 0.06838 & 0.16449 & 0.1728 & 1.000 \\
\hline
\end{tabular}

* All effects have 1 degree of freedom, and residuals have 3,294 degrees of freedom.. 
Table 3. Expected values and linear effect of noise on the difference between PIHA and POKE errors, at fixed STA sizes. Positive values mean greater errors of PIHA. $F$-tests of simple effects (De Rosario, 2013; Fox and Weisberg, 2011), with $p$-values adjusted by Bonferroni's correction.

\begin{tabular}{llrrrr} 
& STA scale & Value & Std. error & $F(1,3294)^{*}$ & p-value \\
\hline Expected error & null & 1.28434 & 0.01446 & 7886.957 & 0.000 \\
difference $(\mathrm{mm})$ & small & 0.56569 & 0.02379 & 565.542 & 0.000 \\
& medium & 0.01183 & 0.03989 & 0.088 & 1.000 \\
& & & & & \\
Difference in & null & 2.6057 & 0.0457 & 3246.433 & 0.000 \\
noise effect & small & 2.1311 & 0.0752 & 802.666 & 0.000 \\
$(\mathrm{~mm} / \mathrm{mm})$ & medium & 1.2354 & 0.1261 & 95.916 & 0.000 \\
\hline
\end{tabular}

* All effects have 1 degree of freedom, and residuals have 3,294 degrees of freedom.

\section{Discussion}

The reported experiment simulated STA-driven marker displacements between 4.5 and $9 \mathrm{~mm}$, and instrumental noise with $\sigma \leq 1 \mathrm{~mm}$, which are normal values in stereophotogrammetry measures (Chiari et al., 2005).

It is known that isotropic marker noise has anisotropic effects on the IHA, whose error has a component dominated by the separation between the IHA and the marker cluster center (Page et al., 2007). Likewise, the CoR was "attracted" by the markers of the arm, and this dislocation could even exceed the size of STA effects with Woltring's original method. Such a systematic bias, even in the presence of purely random error, was approximately proportional to the variance of that error.

The proposed method optimizes a velocity error function that assumes independency of errors across the measure, so it achieved a significant correction of white noise effects. STA, which are the main source of error in human movement analysis (Alexander and Andriacchi, 2001), were not corrected for small noise amplitudes. But the results also showed an interacting effect of noise, which could exceed the size of the "noiseless" STA error. This amplification of STA was reduced, and even removed, with the optimized method and the ranges of noise used in the simulations.

It may be noted that the results were based on an approximation of the gleno-humeral motion: the axes of the local reference frames were not defined in the standard way, and the bone rotation was modeled from the observed skin movement, so the "ideal" position of the CoR and bone motions were not accurate measures, just a reference for 
the simulations. But the most important limitation was that the effect of STA in real measures may be larger than the one obtained in these simulations, since they were modeled upon patterns of marker cluster deformations, whereas the main kinematic effect of real STA are rigid rotations and displacements of the soft tissue, which are greater than deformations (Andersen et al., 2010; De Rosario et al., 2012).

Moreover, it is recommended to place more than three markers for improving precision in the calculation of CoR (Roosen et al., 2013), and higher sampling rates may improve the performance of noise filtering. That combination of factors may do noise effects less noticeable in the presence of real STA than what we obtained in the simulations. For effective reduction of STA errors, it is advisable to take into account the range of motion, velocity, and characteristics of the movement and the artifact itself; depending on those conditions, position-based methods like the FHA pivot or SCoRE may give better results (Monnet et al., 2007; De Rosario et al., 2013)

But focusing on velocity-based methods, like the ISB recommendation, calculating the point of optimal kinematic error instead of the IHA pivot provides a clear benefit in terms of reducing noise effects. Another advantage is that the result of this method has a clear physical meaning (the point with smallest velocity); moreover it does not require intermediate calculations for obtaining IHA positions, which may introduce big numerical errors, nor does it depend on arbitrary thresholds for discarding erroneous data.

\section{Acknowledgements}

This work has been funded by the Spanish Government (Grants DPI2009-13830-C0201, DPI2009-13830-C02-02, DPI2010-20814-C02-01, DPI2010-20814-C02-02).

\section{References}

Alexander, E.J., Andriacchi, T.P., 2001. Correcting for deformation in skin-based marker systems. Journal of Biomechanics 34, 355-361.

Andersen, M.S., Benoit, D.L., Damsgaard, M., Ramsey, D.K., Rasmussen, J., 2010. Do kinematic models reduce the effects of soft tissue artefacts in skin marker-based 
motion analysis? An in vivo study of knee kinematics. Journal of Biomechanics 43, 268-273.

Begon, M., Monnet, T., Lacouture, P., 2007. Effects of movement for estimating the hip joint centre. Gait \& Posture 25, 353-359.

Chiari, L., Croce, U.D., Leardini, A., Cappozzo, A., 2005. Human movement analysis using stereophotogrammetry: Part 2: Instrumental errors. Gait \& Posture 21, $197-211$.

De Rosario, H., 2013. phia: Post-Hoc Interaction Analysis. R package version 0.1-2 URL http://CRAN.R-project.org/package=phia

De Rosario, H., Page, Á., Besa, A., Valera, Á., 2013. Propagation of soft tissue artifacts to the center of rotation: A model for the correction of functional calibration techniques. Journal of Biomechanics 46, 2619-2625.

De Rosario, H., Page, Á., Mata, V., Besa, A., Moreno, R., 2012. Propagation of artifact errors on kinematic variables. Effect on Euler angles. Journal of Biomechanics 45, Supplement 1, S293.

Eaton, J.W., Bateman, D., Hauberg, S., 2008. GNU Octave Manual Version 3. Network Theory Limited.

Ehrig, R.M., Taylor, W.R., Duda, G.N., Heller, M.O., 2006. A survey of formal methods for determining the centre of rotation of ball joints. Journal of Biomechanics 39, 2798-2809.

Fox, J., Weisberg, S., 2011. An R Companion to Applied Regression, Second. ed. Sage, Thousand Oaks CA.

Halvorsen, K., Lesser, M., Lundberg, A., 1999. A new method for estimating the axis of rotation and the center of rotation. Journal of Biomechanics 32, 1221-1227.

Karduna, A.R., McClure, P.W., Michener, L.A., Sennett, B., 2001. Dynamic measurements of three-dimensional scapular kinematics: a validation study. $\mathrm{J}$ Biomech Eng 123, 184-190.

Lanshammar, H., 1982. On precision limits for derivatives numerically calculated from noisy data. Journal of Biomechanics 15, 459-470.

Leardini, A., Cappozzo, A., Catani, F., Toksvig-Larsen, S., Petitto, A., Sforza, V., Cassanelli, G., Giannini, S., 1999. Validation of a functional method for the estimation of hip joint centre location. Journal of Biomechanics 32, 99-103. 
Monnet, T., Desailly, E., Begon, M., Vallée, C., Lacouture, P., 2007. Comparison of the SCoRE and HA methods for locating in vivo the glenohumeral joint centre. Journal of Biomechanics 40, 3487-3492.

Page, Á., Mata, V., Hoyos, J.V., Porcar, R., 2007. Experimental determination of instantaneous screw axis in human motions. Error analysis. Mechanism and Machine Theory 42, 429-441.

Page, Á., Rosario, H. de, Mata, V., Atienza, C., 2009. Experimental Analysis of Rigid Body Motion. A Vector Method to Determine Finite and Infinitesimal Displacements From Point Coordinates. Journal of Mechanical Design 131, 031005 .

R Core Team, 2013. R: A Language and Environment for Statistical Computing. Vienna, Austria.

Roosen, A., Pain, M.T.G., Thouzé, A., Monnet, T., Begon, M., 2013. Segmentembedded frame definition affects the hip joint centre precision during walking. Medical Engineering \& Physics 35, 1228-1234.

Šenk, M., Chèze, L., 2006. Rotation sequence as an important factor in shoulder kinematics. Clinical Biomechanics 21, Supplement 1, S3-S8.

Stokdijk, M., Nagels, J., Rozing, P.M., 2000. The glenohumeral joint rotation centre in vivo. Journal of Biomechanics 33, 1629-1636.

Veeger, H.E.J., Yu, B., 1996. Orientation of axes in the elbow and forearm for biomechanical modelling. In: Biomedical Engineering Conference, 1996., Proceedings of the 1996 Fifteenth Southern. Presented at the Biomedical Engineering Conference, 1996., Proceedings of the 1996 Fifteenth Southern, pp. $377-380$.

Woltring, H.J., 1990. Data processing and error analysis. In: Cappozzo, A., Berme, P. (Eds.), Biomechanics of Human Movement: Applications in Rehabilitation, Sport and Ergonomics. Bertec Corporation, Worthington, OH, pp. 203-227.

Woltring, H.J., Huiskes, R., Lange, A. de, Veldpaus, F.E., 1985. Finite centroid and helical axis estimation from noisy landmark measurements in the study of human joint kinematics. Journal of Biomechanics 18, 379-389.

Wu, G., Cavanagh, P.R., 1995. ISB recommendations for standardization in the reporting of kinematic data. Journal of Biomechanics 28, 1257-1261. 
Wu, G., van der Helm, F.C.T., (DirkJan) Veeger, H.E.J., Makhsous, M., Van Roy, P., Anglin, C., Nagels, J., Karduna, A.R., McQuade, K., Wang, X., Werner, F.W., Buchholz, B., 2005. ISB recommendation on definitions of joint coordinate systems of various joints for the reporting of human joint motion-Part II: shoulder, elbow, wrist and hand. Journal of Biomechanics 38, 981-992.

\section{Appendix}

\section{Appendix A1. Mathematical proof of the optimized method}

The method proposed in this paper optimizes the kinematic error at the CoR. If the reference point $\mathbf{p}_{t}$ is set at the origin of coordinates for any $t$, the velocity of any fixed point $\mathbf{s}$ will be:

$$
\dot{\mathbf{s}}=\dot{\mathbf{p}}_{t}+\boldsymbol{\omega}_{t} \times \mathbf{s}
$$

or in matrix form:

$$
\dot{\mathbf{s}}=\dot{\mathbf{p}}_{t}+\left(\begin{array}{ccc}
0 & -\omega_{z t} & \omega_{y t} \\
\omega_{z t} & 0 & -\omega_{x t} \\
-\omega_{y t} & \omega_{x t} & 0
\end{array}\right) \mathbf{S}=\dot{\mathbf{p}}_{t}+\mathbf{A}_{t} \mathbf{S}
$$

The velocity at the CoR should be null, so the function that must be optimized is:

$$
f=\sum_{t=1}^{n}\left|\dot{\mathbf{p}}_{t}+\mathbf{A}_{t} \mathbf{S}\right|^{2}=\sum_{t=1}^{n} \dot{\mathbf{p}}_{t}^{T} \dot{\mathbf{p}}_{t}+2 \dot{\mathbf{p}}_{t} \mathbf{A}_{t} \mathbf{S}+\mathbf{s}^{T} \mathbf{A}_{t}^{T} \mathbf{A}_{t} \mathbf{S}
$$

The point $\mathbf{s}_{\text {POKE }}$ that satisfies this condition is:

$$
\frac{\partial f}{\partial \mathbf{s}}=2\left(\sum_{t=1}^{n} \mathbf{A}_{t}^{T} \dot{\mathbf{p}}_{t}+\mathbf{A}_{t}^{T} \mathbf{A}_{t} \mathbf{s}\right)=0
$$

Since $\mathbf{A}_{t}^{T}=-\mathbf{A}_{t}$, and $\mathbf{A}_{t}^{T} \mathbf{A}_{t}=\mathbf{W}_{t}$ as defined in (5), equation (A1.4) is just equivalent to $(6)$.

\section{Appendix A2. Continuous noise model}

The error added to the marker coordinates was based on the model used by Begon et al. (2007). For each marker $i$, coordinate $j$, and gesture $k$ (flexion-extension, elevation, 
circumduction), there was a fixed coefficient $a 2_{i, j, k}$ (the underlined values of table 1) that modeled the "continuous" part of the error (simulated STA), such that it was minimal when the rotation angle $\theta_{t}$ was null (around the reference posture), and maximal when its absolute value approached the upper limit $\theta_{k}^{M}$. Another set of random coefficients was used to vary the STA patterns, and add white noise, according to the following error function:

$$
N_{i, j, k, t}=A \cdot a 1 \cdot a 2_{i, j, k} \cdot a 3 \exp \left(-\frac{\left(\theta_{t} \mid-\theta_{k}^{M} b\right)^{2}}{2 \theta_{k}^{M} c^{2}}\right)+\sigma \cdot d,(\mathrm{~A} 2.1)
$$

$a 1$ alternated randomly between $\{-1 ; 1\} . a 3, b, c$ were uniformly distributed in the ranges $a 3 \in[0.9,1], b \in[0.85,1], c \in[0.1,0.3]$, and $d$ was distributed as a standard normal. $\theta_{t}$ was the main angle of each gesture, according to the XZY Euler sequence recommended by Šenk and Chèze (2006): flexion-extension for the flexion and circumduction gestures (upper limit $\theta_{k}^{M}=45^{\circ}$ ), and abduction-adduction for elevation $\left(\theta_{k}^{M}=35^{\circ}\right)$. Finally, $A$ and $\sigma$ were the scaling factors for the artifact and noise, respectively. 